\title{
Waveform domain framework for capacity analysis of uplink WCDMA systems
}

\author{
Tan Tai Do ${ }^{1}$, Su Min Kim²* ${ }^{*}$, Tobias J. Oechtering ${ }^{1}$, Mikael Skoglund ${ }^{1}$ and Gunnar Peters ${ }^{3}$
}

\begin{abstract}
This paper investigates the capacity limit of an uplink WCDMA system considering a continuous-time waveform signal. Various realistic assumptions are incorporated into the problem, which make the study valuable for performance assessment of real cellular networks to identify potentials for performance improvements in practical receiver designs. An equivalent discrete-time channel model is derived based on sufficient statistics for optimal decoding of the transmitted messages. The capacity regions are then characterized using the equivalent channel considering both finite constellation and Gaussian distributed input signals. The capacity with sampling at the receiver is also provided to exemplify the performance loss due to a typical post-processing at the receiver. Moreover, we analyze the asymptotic capacity when the signal-to-noise ratio goes to infinity. The conditions to simultaneously achieve the individual capacities are derived, which reveal the impacts of signature waveform space, channel frequency selectivity and signal constellation on the system performance.
\end{abstract}

Keywords: Uplink WCDMA, Capacity analysis, Continuous-time, Waveform domain, Time-variant spreading, Sampling, Finite constellation

\section{Introduction}

Code division multiple access (CDMA) has become standard in several wireless communication systems from IS-95, UMTS wideband CDMA (WCDMA) to HSPA, and so on [1-3]. Although being introduced more than 50 years ago, CDMA is still largely employed and developed nowadays due to its various advantages such as enabling universal frequency reuse, improving handover performance by soft-handover, and mitigating the effects of interference and fading. The performance assessment of such networks is of significant importance. In addition, the architecture of WCDMA systems still has room for improvement, especially at the uplink receiver side (base station) $[4,5]$.

In the literature, most studies on fundamental limits of multiuser CDMA systems have been done under the assumptions of synchronous, time-invariant (each user

\footnotetext{
*Correspondence: suminkim@kpu.ac.kr

This work was partly presented at the 80th IEEE Vehicular Technology Conference (VTC2014-Fall), Vancouver, Canada, September 2014.

2 Department of Electronics Engineering, Korea Polytechnic University, 15073

237 Sangidaehak-ro, Gyeonggi-do, Korea

Full list of author information is available at the end of the article
}

uses the same spreading sequence for all data symbols), and/or random spreading sequences [6-11]. In [6-8], the optimal spreading sequences and capacity limits for synchronous CDMA have been studied with a discretetime signal model. A more theoretical approach on CDMA capacity analysis has been pursued in [9-11] by modeling the spreading sequences with random sequences. However, the assumption of perfect synchronization between users is not realistic, especially for a cellular CDMA uplink. Moreover, in practice, timevariant-spreading sequences based on Gold or Kasami codes [1-3] are often used rather than time-invariant or random spreading sequences.

The capacity limit for a CDMA system with symbolasynchronous transmission (the symbol epochs of the signal are not aligned at the receiver) has also been studied in [12-15]. In [12], Verdú studied the capacity region of an uplink time-invariant CDMA system with inter-symbol interference (ISI) by exploiting the asymptotic properties of Toeplitz matrices. In $[13,14]$, the authors studied user and sum capacities of a symbol-asynchronous CDMA system but with chip-synchronous transmission (the timing of the chip epochs are aligned) assumption, which made

\section{是 Springer}

(C) 2015 Do et al. Open Access This article is distributed under the terms of the Creative Commons Attribution 4.0 International License (http://creativecommons.org/licenses/by/4.0/), which permits unrestricted use, distribution, and reproduction in any medium, provided you give appropriate credit to the original author(s) and the source, provide a link to the Creative Commons license, and indicate if changes were made. 
the analysis tractable using a discrete-time model. In [15], the spectral efficiency of an asynchronous CDMA system has been considered while neglecting the ISI by assuming a large spreading factor.

There have been several studies trying to deal with the continuous-time asynchronous CDMA system setup. However, most of them focus on other performance metrics than capacity (e.g., error probability considering different detection algorithms) [16-20]: time-invariant CDMA [21] or asynchronous CDMA but with an ISIfree assumption [22]. The capacity analysis for a real cellular network with continuous-time waveform, timevariant-spreading, asynchronous CDMA is difficult due to the following reasons. First, an equivalent discretetime signal model is complicated to be expressed due to the asynchronization between symbols and chips. Next, for a time-variant spreading CDMA system, the approach based on the asymptotic properties of a Toeplitz form [23], which is crucial for the capacity analysis with ISI channel $[12,24]$, cannot be employed since the variation of spreading sequence destroys the Toeplitz structure of the equivalent channel matrix.

\subsection{Contributions of this work}

Motivated by the fact that most existing research on multiuser CDMA capacity have focused on theoretical analysis with simplified system assumptions, in this work, we present a framework for capacity analysis of a WCDMA system with more realistic assumptions, which make the framework and results more valuable for the performance assessment of real cellular networks. Our main contributions are summarized as follows:

- We provide a precise channel model reflecting practical operations of the uplink WCDMA physical (PHY) layer based on the 3GPP release 11 specification [1]. Various realistic assumptions are included into the system such as: continuous-time waveform-transmitted signal and time-variant spreading and an asynchronous multicode CDMA system with ISI over frequency-selective channels. It is worth noting that although the signal model is constructed based on a WCDMA system, the approach and framework can be extended or transferred to other wireless standards.

- We derive sufficient statistics for decoding the transmitted symbols based on the continuous-time-received signal, which provides us an equivalent discrete-time signal model. A matrix representation of channel model is provided for which the equivalent additional noise is shown to be a Gaussian distributed random vector.

- Since sufficient statistics preserve the mutual information ([25], Chap. 2), the capacity is then derived using the equivalent discrete-time signal model. In particular, we characterize the capacity region when the input signal is fixed to finite constellations, e.g., PSK, QAM, and so on, with a uniform input distribution, which are widely used in current real cellular networks. Additionally, we provide the capacity region when the input signal follows a Gaussian distribution, which is the optimal input distribution for additive Gaussian noise channels. Accordingly, the Gaussian capacity offers a capacity outer bound for the real WCDMA cellular networks using finite constellation input.

- Due to the data-processing inequality ([25], Chap. 2), the mutual information between input and output cannot increase through any post-processing at the receiver. Given the capacity bounds measured directly at the receive antenna of a real system, we can now assess the capacity loss due to a specific post-processing at the receiver. Therewith, we investigate the capacity loss due to sampling, which is a traditional discretization approach in practical systems. Note that in the real cellular networks, since the sampling window is finite, perfect reconstruction of a band-limited signal is not guaranteed even if the sampling rate is equal to Nyquist rate ([26], Chap. 8). The assessment of such impact on the capacity is also considered in this work.

- We analyze the asymptotic sum-capacity when the signal-to-noise ratio (SNR) goes to infinity, for which we derive the conditions on the signature waveform space so that on every link to the base station, the individual capacities are achieved simultaneously. To this end, we first derive the sufficient condition, which holds for all kinds of input signals including signals based on finite and infinite constellations. Next, once again, we motivate our study from a practical perspective by focusing on the finite constellation input signal. Accordingly, a necessary condition to simultaneously achieve the individual capacities with a finite constellation input signal, which takes the signal constellation structure into account, is derived. Those results are particularly useful for spreading sequence design in a real WCDMA cellular network.

The rest of the paper is organized as follows: Section 2 presents the signal model where sufficient statistics and an equivalent matrix representation are derived. In Section 3, the capacity analysis is provided considering finite constellations and Gaussian-distributed input signals. The capacity employing sampling is also investigated in this section. The asymptotic capacity when the SNR goes to infinity is analyzed and discussed in Section 4. Finally, Section 5 concludes the paper.

\section{Signal model}

Since the physical layer defines the fundamental capacity limit of the uplink WCDMA channel [2], we focus on a signal model reflecting the operations of the uplink 
WCDMA PHY layer based on the 3GPP release 11 specification [1].

\subsection{Waveform signal model}

Let us consider a $K$-user multi-code WCDMA system with $M$ codes for each I/Q branch and spreading factor $N_{s f}$. Then, the transmitted signal for user $k$ can be expressed as

$$
x_{k}(t)=\sqrt{E_{k}} \sum_{i=1}^{N} \sum_{m=1}^{M} d_{k i}^{m} s_{k i}^{m}(t), k=1, \ldots, K,
$$

where $E_{k}$ denotes the transmitted power of user $k, N$ denotes the number of symbols, $s_{k i}^{m}(t)=\frac{1}{\sqrt{N_{s f}}} \sum_{n=0}^{N_{s f}-1}$ $c_{k i}^{m}[n] p\left(t-(i-1) T_{s}-n T_{c}\right)$ is the signature waveform for the $i$-th symbol of the $m$-th stream of user $k, p(t)$ is the chip waveform with unit power and finite bandwidth $W^{1}, T_{c}$ is the chip duration and $T_{s}=N_{s f} T_{c}$ is the symbol duration, and $c_{k i}^{m}[n]$ denotes the spreading sequence which satisfies $\sum_{n=0}^{N_{s f}-1}\left|c_{k i}^{m}[n]\right|^{2}=N_{s f}$. In a time-variant CDMA system, a different spreading sequence $\left\{c_{k i}^{m}[n]\right\}_{n}$ is used for each transmitted symbol $d_{k i}^{m}$. This corresponds to a real cellular CDMA network with long scrambling codes, in which the effective spreading sequence will vary between symbols.

In this study, we assume a tapped-delay line channel model $^{2}$ with $L$ multi-paths ([27] Chap. 2), i.e.,

$$
h_{k}(t)=\sum_{l=1}^{L} g_{k l} \delta\left(t-\tau_{k l}\right), k=1, \ldots, K,
$$

where $g_{k l}$ and $\tau_{k l}$ denote the channel coefficient and the propagation delay for the $l$-th path of the channel for user $k$, respectively. Then the received signal is given by

$$
\begin{aligned}
r(t) & =\sum_{k=1}^{K} h_{k}(t) * x_{k}\left(t-\lambda_{k}\right)+n(t) \\
& =\sum_{k=1}^{K} \sum_{i=1}^{N} \sum_{m=1}^{M} \sqrt{E_{k}} d_{k i}^{m} \sum_{l=1}^{L} g_{k l} s_{k i}^{m}\left(t-\lambda_{k}-\tau_{k l}\right)+n(t),
\end{aligned}
$$

where $\lambda_{k}$ denotes the time delay of the transmitted signal from user $k$, the symbol $*$ denotes the convolution operation, and $n(t)$ represents the additive white Gaussian noise with a two-sided power spectral density (PSD) $N_{0} / 2=\sigma^{2}$.

\subsection{Sufficient statistic and equivalent channel}

Since a sufficient statistic for decoding the transmitted messages preserves the capacity of the system, the capacity of a continuous-time channel can be computed using a sufficient statistic ([25], Chap. 2), ([28], Chap. 8). To this end, let us define the transmitted symbol vectors $\mathbf{d}_{k i}:=\left[d_{k i}^{1}, \ldots, d_{k i}^{M}\right]^{T} \in \mathbb{C}^{M \times 1}$ (for each stream), $\mathbf{d}_{k}:=$ $\left[\mathbf{d}_{k 1}{ }^{T}, \ldots, \mathbf{d}_{k N}{ }^{T}\right]^{T} \in \mathbb{C}^{N M \times 1}$ (for each user), and $\mathbf{d}:=$ $\left[\mathbf{d}_{1}{ }^{T}, \ldots, \mathbf{d}_{K}{ }^{T}\right]^{T} \in \mathbb{C}^{K N M \times 1}$ (for all users), where $(\cdot)^{T}$ denotes the transpose operation. Further, let us define $\mu(t ; \mathbf{d})$ as the received signal without noise, i.e.,

$\mu(t ; \mathbf{d}):=\sum_{k=1}^{K} \sum_{i=1}^{N} \sum_{m=1}^{M} \sqrt{E_{k}} d_{k i}^{m} \sum_{l=1}^{L} g_{k l} s_{k i}^{m}\left(t-\lambda_{k}-\tau_{k l}\right)$.

The problem of optimal decoding $\mathbf{d}$ is similar to the detection problem in ([27], Proposition 3.2) (see [18] for a similar approach based on the Cameron-Martin formula [29], Chap. VI). Accordingly, the optimal decision ${ }^{3}$ can be made using the following decision variables

$$
\Phi(\mathbf{d})=2 \Re\left\{\int_{-\infty}^{\infty} \mu(t ; \mathbf{d})^{*} r(t) d t\right\}-\int_{-\infty}^{\infty}[\mu(t ; \mathbf{d})]^{2} d t
$$

where $\mathfrak{R}\{\cdot\}$ denotes the real part of a complex value and $(\cdot)^{*}$ denotes the complex conjugate operation. Since the second term of (3) does not depend on the received signal $r(t)$, we can drop it. Therewith, the sufficient statistic is based on the first term of (3), which can be rewritten as

$$
2 \Re\left\{\sum_{k=1}^{K} \sum_{i=1}^{N} \sum_{m=1}^{M} \sqrt{E_{k}} d_{k i}^{m *} \sum_{l=1}^{L} g_{k l} * \int_{-\infty}^{\infty} r(t) s_{k i}^{m}\left(t-\lambda_{k}-\tau_{k l}\right)^{*} d t\right\} .
$$

Let us denote $y_{k i}^{m l}:=\int_{-\infty}^{\infty} r(t) s_{k i}^{m}\left(t-\lambda_{k}-\tau_{k l}\right)^{*} d t$ and $z_{k i}^{m}:=\sum_{l=1}^{L} g_{k l} y_{k i}^{m l}$, then $\left\{z_{k i}^{m}\right\}_{k, i, m}$ is a sufficient statistic for decoding $\mathbf{d}$ based on $r(t)$. It is shown that the received signal passing through a bank of matched filters, where the received signal is matched to the delayed versions of the signature waveforms, results in a sufficient statistic for decoding d based on $r(t)$. Figure 1 illustrates an implementation to obtain the sufficient statistic from the continuous-time received signal. This has a RAKE receiver structure ([30], Chap. 14), including RAKE-matched fingers followed by maximal ratio combining (MRC).

Moreover, let $\rho_{(\mathrm{kiml})}^{\left(k^{\prime} i^{\prime} \mathrm{m}^{\prime} l^{\prime}\right)}$ be the cross-correlation function between the signature waveforms defined as

$$
\begin{aligned}
\rho_{(k i m l)}^{\left(k^{\prime} i^{\prime} m^{\prime} l^{\prime}\right)}= & \int_{-\infty}^{\infty} s_{k i}^{m}\left(t-\lambda_{k}-\tau_{k l}\right)^{*} s_{k^{\prime} i^{\prime}}^{m^{\prime}}\left(t-\lambda_{k^{\prime}}-\tau_{k^{\prime} l^{\prime}}\right) d t \\
= & \frac{1}{N_{s f}} \sum_{n=0}^{N_{s f}-1} \sum_{n^{\prime}=0}^{N_{s f}-1} c_{k i}^{m}[n]^{*} c_{k^{\prime} i^{\prime}}^{m^{\prime}}\left[n^{\prime}\right] \\
& \times R_{p}\left(\frac{n-n^{\prime}}{N_{s f}} T_{s}+\left(i-i^{\prime}\right) T_{s}+\left(\tau_{k l}-\tau_{k^{\prime} l^{\prime}}\right)+\left(\lambda_{k}-\lambda_{k^{\prime}}\right)\right),
\end{aligned}
$$

where $R_{p}(\tau)=\int_{-\infty}^{\infty} p(t)^{*} p(t+\tau) d t$ is the autocorrelation function of the chip waveform. Then the sufficient statistics can be expressed as

$$
z_{k i}^{m}=\sum_{l=1}^{L} \sum_{k^{\prime}=1}^{K} \sum_{i^{\prime}=1}^{N} \sum_{m^{\prime}=1}^{M} \sum_{l^{\prime}=1}^{L} \sqrt{E_{k^{\prime}}} d_{k^{\prime} i^{\prime}}^{m^{\prime}} g_{k l}{ }^{*} g_{k^{\prime} l^{\prime}} \rho_{(k i m l)}^{\left(k^{\prime \prime} m^{\prime} l^{\prime}\right)}+n_{k i}^{m},
$$




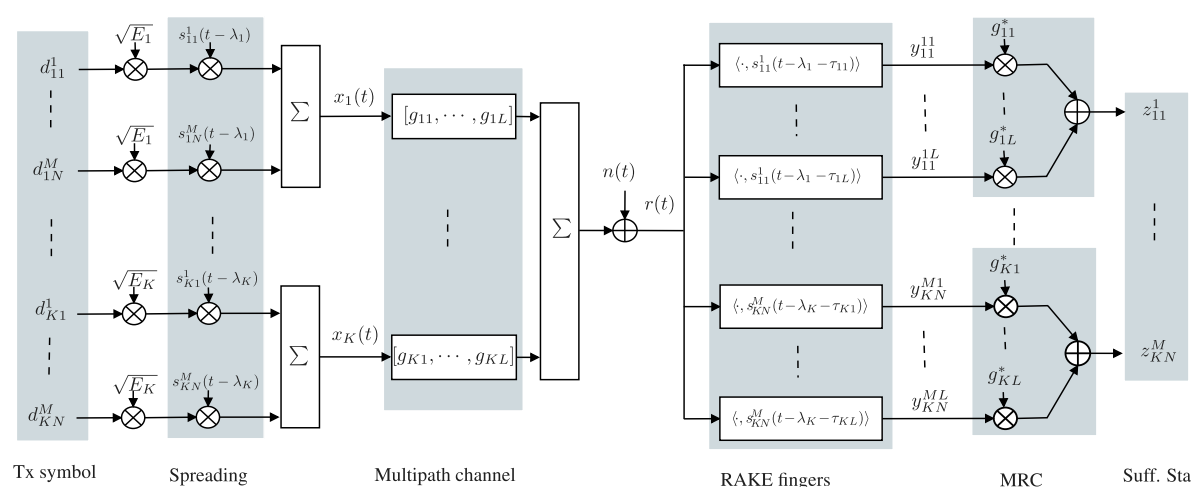

Fig. 1 Diagram of the implementation to obtain the sufficient statistic from the continuous-time received signal of a K-user uplink WCDMA system with $M$ codes for each I/Q branch over an L-tap frequency-selective channel

where $n_{k i}^{m}:=\sum_{l=1}^{L} g_{k l} * \int_{-\infty}^{\infty} n(t) s_{k i}^{m}\left(t-\lambda_{k}-\tau_{k l}\right)^{*} d t$ is the equivalent noise term associated with $z_{k i}^{m}$ after matched filtering.

A matrix canonical form is useful to characterize the capacity from a sufficient statistic. Hence, we express the sufficient statistics $\left\{z_{k i}^{m}\right\}_{k, i, m}$ derived in (4) as an equivalent matrix equation. By following the similar steps as in [31], the matrix representation of the equivalent channel can be obtained from (4) as

$$
\mathbf{z}=\sum_{k=1}^{K} \sqrt{E_{k}} \mathbf{H}_{k} \mathbf{d}_{k}+\mathbf{n},
$$

where $\mathbf{z}:=\left[z_{11}^{1}, \cdots, z_{K N}^{M}\right]^{T} \in \mathbb{C}^{K N M \times 1}, \mathbf{d}_{k}:=$ $\left[d_{k 1}^{1}, \cdots, d_{k N}^{M}\right]^{T} \in \mathbb{C}^{N M \times 1}$, and $\mathbf{n}:=\left[n_{11}^{1}, \cdots, n_{K N}^{M}\right]^{T} \in$ $\mathbb{C}^{K N M \times 1}$. The equivalent channel channel $\mathbf{H}_{k}$ is given by

$$
\mathbf{H}_{k}=\left[\begin{array}{c}
\mathbf{G}_{1}^{\dagger} \mathbf{R}_{k}^{1} \mathbf{G}_{k} \\
\vdots \\
\mathbf{G}_{K}^{\dagger} \mathbf{R}_{k}^{K} \mathbf{G}_{k}
\end{array}\right] \in \mathbb{C}^{K N M \times N M},
$$

where the channel gain matrix $\mathbf{G}_{k}$ is block diagonal and given by

$$
\mathbf{G}_{k}=\operatorname{blkdiag}(\underbrace{\mathbf{g}_{k}, \cdots, \mathbf{g}_{k}}_{M N \text { vectors }}) \in \mathbb{C}^{L N M \times N M}
$$

with $\mathbf{g}_{k}=\left[g_{k 1}, \cdots, g_{k L}\right]^{T} \in \mathbb{C}^{L \times 1}$, and the correlation matrix is defined as

$$
\mathbf{R}_{k}^{k^{\prime}}=\left[\begin{array}{ccccc}
\rho_{(k 111)}^{\left(k^{\prime} 111\right)} & \cdots & \rho_{(k 111)}^{\left(k^{\prime} 11 L\right)} & \cdots & \rho_{(k 111)}^{\left(k^{\prime} N M L\right)} \\
\rho_{(k 112)}^{\left(k^{\prime} 111\right)} & \cdots & \rho_{(k i 12)}^{\left(k^{\prime} i^{\prime} 1 L\right)} & \cdots & \rho_{(k 112)}^{\left(k^{\prime} N M L\right)} \\
\vdots & \vdots & \vdots & \vdots & \vdots \\
\rho_{(k N M L)}^{\left(k^{\prime} 111\right)} & \cdots & \rho_{(k N M L)}^{\left(k^{\prime} 11 L\right)} & \cdots & \rho_{(k N M L)}^{\left(k^{\prime} N M L\right)}
\end{array}\right] \in \mathbb{C}^{N M L \times N M L} .
$$

Moreover, it is shown in Appendix 1 that the equivalent noise vector $\mathbf{n}$ is a complex Gaussian random vector with zero mean and covariance matrix $\sigma^{2} \mathbf{H}$ with $\mathbf{H}=$ $\left[\mathbf{H}_{1}, \ldots, \mathbf{H}_{K}\right] \in \mathbb{C}^{K N M \times K N M}$.

Remark 1 . In this work, the signal model is constructed based on the practical operation of an uplink WCDMA PHY layer. However, the approach and framework can be extended or transferred to other wireless standards. Indeed, the signal model in (1) can be used to describe the continuous-time-transmitted signal of a general system, in which $s_{k i}^{m}(t)$ are the waveforms used for the modulation at the transmitter. For example, in a OFDM system, $s_{k i}^{m}(t)$ can be replaced by the corresponding orthogonal waveforms. Moreover, the resulting equivalent channel in (5) corresponds to a traditional discrete-time MIMO multiple-access channel (MAC), which are used in various research literature.

\section{Capacity analysis}

In this section, we analyze the capacity of the continuoustime uplink WCDMA channel. Recalling that $\mathbf{z}$ is a sufficient statistic for optimal (i.e., capacity preserving) decoding $\mathbf{d}$ based on $r(t)$. Any coding scheme which achieves the capacity of the channel with input $\mathbf{d}$ and output $r(t)$ can also be employed to the channel with input $\mathbf{d}$ and decoding based on output $\mathbf{z}$ instead of $r(t)$. Therefore, the channel capacity is preserved when the continuous-time output $r(t)$ is replaced by the sufficient statistic $\mathbf{z}$. Thus, we can focus on the capacity of the equivalent discretetime channel in (5), which is given by the capacity region of a discrete memoryless MAC [32].

Let us define $R_{1}, R_{2}, \ldots, R_{K}$ as the maximum number of bits that can be reliably transmitted from user 1 , user $2, \ldots$, user $K$ per block of $N$ symbols. The capacity region of the uplink WCDMA channel is then characterized by the closure of the convex hull of the union of all achievable 
rate vectors $\left(R_{1}, R_{2}, \ldots, R_{K}\right)$ satisfying [32], ([25], Chapter 15)

$$
\sum_{k \in \mathcal{J}} R_{k} \leq I\left(\mathbf{d}_{\mathcal{J}} ; \mathbf{z} \mid \mathbf{d}_{\mathcal{J}^{c}}\right)
$$

for all index subsets $\mathcal{J} \subseteq\{1, \ldots, K\}$ and some joint pmf $p(\mathbf{d})=\prod_{k=1}^{K} p\left(\mathbf{d}_{k}\right)$, where $\mathcal{J}^{c}$ denotes the complement of $\mathcal{J}$ and $\mathbf{d}_{\mathcal{J}}=\left\{\mathbf{d}_{k}: k \in \mathcal{J}\right\}$.

We now characterize the uplink WCDMA capacity region considering two specific input signals: finite constellation with uniformly distributed input and Gaussiandistributed input.

\subsection{Finite constellation input}

When the input signal vector $\mathbf{d}_{k}$ at each user is independently taken from a finite constellation set $\mathcal{M}^{N M}$, $|\mathcal{M}|=M_{c}$, with equal probability, i.e., $p\left(\mathbf{d}_{k}\right)=\frac{1}{M_{c}^{N M}}$, $\forall k \in\{1, \ldots, K\}$, then the rate constraints in (7) can be rewritten as

$$
\begin{aligned}
\sum_{k \in \mathcal{J}} R_{k} & \leq I\left(\mathbf{d}_{\mathcal{J}} ; \mathbf{z} \mid \mathbf{d}_{\mathcal{J}^{c}}\right) \\
& =h\left(\mathbf{z} \mid \mathbf{d}_{\mathcal{J}^{c}}\right)-h\left(\mathbf{z} \mid \mathbf{d}_{\mathcal{J}^{\prime}} \mathbf{d}_{\mathcal{J}^{c}}\right)=h\left(\mathbf{z}_{\mathcal{J}}\right)-h(\mathbf{n}) \\
& =-\mathbb{E}\left\{\log _{2}\left(f_{\mathbf{z}_{\mathcal{J}}}(\bar{z})\right)\right\}-\log _{2}\left(\operatorname{det}\left(\pi e \sigma^{2} \mathbf{H}\right)\right)
\end{aligned}
$$

for all $\mathcal{J} \subseteq\{1, \ldots, K\}$ with $\mathbf{z}_{\mathcal{J}}:=\sum_{k \in \mathcal{J}} \sqrt{E_{k}} \mathbf{H}_{k} \mathbf{d}_{k}+\mathbf{n}$. $\mathbf{z}_{\mathcal{J}}$ is a Gaussian mixture random vector with probability density function (pdf)

$$
f_{\mathbf{z}_{\mathcal{J}}}(\bar{z})=\sum_{\bar{d} \in \mathcal{M}|\mathcal{J}| N M} p\left(\mathbf{d}_{\mathcal{J}}=\bar{d}\right) \cdot f_{\mathbf{z}_{\mathcal{J}} \mid \mathbf{d}_{\mathcal{J}}}(\bar{z} \mid \bar{d})
$$

where $p\left(\mathbf{d}_{\mathcal{J}}=\bar{d}\right)=\frac{1}{M_{c}^{|\mathcal{J}| N M}}$ and $f_{\mathbf{z}_{\mathcal{J}} \mid \mathbf{d}_{\mathcal{J}}}(\bar{z} \mid \bar{d})$ is the conditional pdf of $\mathbf{z}_{\mathcal{J}}$ given $\mathbf{d}_{\mathcal{J}}$. Let us denote $\mathbf{E}_{\mathcal{J}} \mathbf{H}_{\mathcal{J}} \mathbf{d}_{\mathcal{J}}:=$ $\sum_{k \in \mathcal{J}} \sqrt{E_{k}} \mathbf{H}_{k} \mathbf{d}_{k}$, where $\mathbf{E}_{\mathcal{J}}$ is the power scaled matrix $\mathbf{E}_{\mathcal{J}}:=\operatorname{blkdiag}\left(\left\{\sqrt{E_{k}} \mathbf{I}_{N M}\right\}_{k \in \mathcal{J}}\right)$ and $\mathbf{H}_{\mathcal{J}}$ is the sub-matrix of $\mathbf{H}$ after removing $\mathbf{H}_{k^{\prime}}, \forall k^{\prime} \in \mathcal{J}^{c}$. Then, $f_{\mathbf{z}_{\mathcal{J}} \mid \mathbf{d}_{\mathcal{J}}}(\bar{z} \mid \bar{d})$ is the pdf of a complex Gaussian random vector with mean $\mathbf{E}_{\mathcal{J}} \mathbf{H}_{\mathcal{J}} \bar{d}$ and covariance matrix $\sigma^{2} \mathbf{H}$, i.e.,

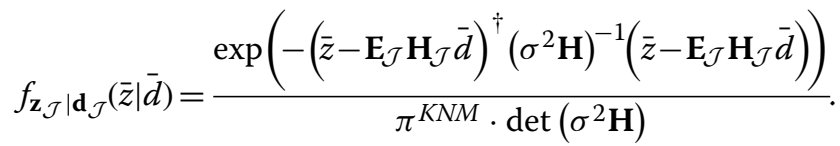

Typically, the capacity region of a channel with finite constellation input is numerically characterized via Monte Carlo simulation because a closed-form expression does not exist. It is worth noting that in order to calculate the first term of (8), one has to average overall possible $M_{c}^{|\mathcal{J}| N M}$ input symbols (up to $M_{c}^{K N M}$ for sum-rate) according to (9). However, when $M_{c}$ and/or $N$ are too large, this task becomes intractable due to prohibitive computational complexity. In MIMO channels with finite constellation input, a similar problem occurs when the input alphabet set or the number of antennas is too large, e.g., 64-QAM or
$8 \times 8$ MIMO [33]. In order to tackle this problem, we have proposed an effective approximation algorithm based on sphere-decoding approach to find the approximate capacity for large MIMO system with finite constellation input in [34]. The algorithm to compute the entropy is out of the scope of this work. However, we use it in the numerical results section (Section 3.4) to compute approximations on the capacity curves for large $N$. The specific details about the algorithm can be found in [34].

\subsection{Gaussian input}

If the input signal vector $\mathbf{d}_{k}$ of each user follows a zero mean complex Gaussian distribution with unit input power constraint, i.e., $\mathbf{d}_{k} \sim \mathcal{C} \mathcal{N}\left(\mathbf{0}, \mathbf{I}_{N M}\right), \forall k=1, \ldots, K$, then the capacity region is characterized by the rate vectors $\left(R_{1}, R_{2}, \ldots, R_{K}\right)$ satisfying

$$
\sum_{k \in \mathcal{J}} R_{k} \leq \log \operatorname{det}\left(\mathbf{I}_{N M}+\sum_{k \in \mathcal{J}} \frac{E_{k}}{\sigma^{2}} \mathbf{H}_{k}^{\dagger} \mathbf{H}^{-1} \mathbf{H}_{k}\right)
$$

for all $\mathcal{J} \subseteq\{1, \ldots, K\}$. Since the Gaussian-distributed input is the optimal input for a given mean power constraint, (10) serves as an outer bound for the capacity region with a practically motivated input, i.e., finite constellation input as discussed in Section 3.1.

\subsection{Sampling}

Since the matched filtering at the receiver yields a sufficient statistic, the uplink WCDMA capacity achieved by any other receiver structures is upper bounded by the capacity achieved by the sufficient statistic using matched filtering. Regarding the capacity upper bounds in Sections 3.1-3.2 as benchmarks for the performance assessment, we now analyze the capacity achieved by sampling to evaluate the capacity performance loss due to specific post-processing at the receiver.

For sampling at the receiver, we assume that out-of-band noise is first suppressed by an ideal low-pass filter (LPF) with bandwidth $W$, which has the same bandwidth as the transmitted signal. Then, the received signals are uniformly sampled at every time instance $t_{n}, n=1, \ldots, N_{\mathrm{sp}}$, where $N_{\mathrm{sp}}$ is finite. As a result, the sampled received signal at time $t_{n}$ is given by

$$
\begin{array}{r}
r_{n}:=r_{\mathrm{lp}}\left(t_{n}\right)=\sum_{k=1}^{K} \sum_{m=1}^{M} \sum_{i=1}^{N} d_{k i}^{m} \sum_{l=1}^{L} g_{k l} s_{\mathrm{lp}, k i}^{m}\left(t_{n}-\lambda_{k}-\tau_{k l}\right) \\
+n_{\mathrm{lp}}\left(t_{n}\right), \quad n=1, \ldots, N_{\mathrm{sp}}
\end{array}
$$

where $r_{\mathrm{lp}}(t), s_{\mathrm{lp}, k i}^{m}(t)$, and $n_{\mathrm{lp}}(t)$ denotes the outputs of $r(t)$, $s_{k i}^{m}(t)$, and $n(t)$ passing through the LPF, respectively. We have $s_{\mathrm{lp}, k i}^{m}(t)=s_{k i}^{m}(t)$ since the ideal LPF is assumed to have the same bandwidth as the transmitted signal, i.e., bandwidth of $s_{k i}^{m}(t)$. Let us denote the effective signature 
waveform by $\bar{s}_{k i}^{m}(t):=\sum_{l=1}^{L} g_{k l} s_{k i}^{m}\left(t-\lambda_{k}-\tau_{k l}\right)$, then the sampled received signal can be expressed as

$$
r_{n}=\sum_{k=1}^{K} \sum_{m=1}^{M} \sum_{i=1}^{N} \sqrt{E_{k}} \bar{s}_{k i}^{m}\left(t_{n}\right) d_{k i}^{m}+n_{\mathrm{lp}}\left(t_{n}\right), n=1, \ldots, N_{\mathrm{sp}} .
$$

Next, let us denote the sampling signature waveform matrix corresponding to user $k$ by

$$
\overline{\mathbf{S}}_{k}=\left[\begin{array}{cccc}
\bar{s}_{k 1}^{1}\left(t_{1}\right) & \bar{s}_{k 1}^{2}\left(t_{1}\right) & \cdots & \bar{s}_{k N}^{M}\left(t_{1}\right) \\
\bar{s}_{k 1}^{1}\left(t_{2}\right) & \bar{s}_{k 1}^{2}\left(t_{2}\right) & \cdots & \bar{s}_{k N}^{M}\left(t_{2}\right) \\
\vdots & \vdots & \vdots & \vdots \\
s_{k 1}^{1}\left(t_{N_{\mathrm{sp}}}\right) & s_{k 1}^{2}\left(t_{N_{\mathrm{sp}}}\right) & \cdots & s_{k N}^{M}\left(t_{N_{\mathrm{sp}}}\right)
\end{array}\right] \in \mathbb{C}^{N_{\mathrm{sp}} \times N M},
$$

and the sampled received signal and sampled noise vectors by

$$
\begin{aligned}
& \mathbf{r}_{\mathrm{sp}}=\left[r_{1}, r_{2}, \cdots, r_{N_{\mathrm{sp}}}\right]^{T} \in \mathbb{C}^{N_{\mathrm{sp}} \times 1}, \\
& \mathbf{n}_{\mathrm{sp}}=\left[n_{\mathrm{lp}}\left(t_{1}\right), n_{\mathrm{lp}}\left(t_{2}\right), \ldots, n_{\mathrm{lp}}\left(t_{N_{\mathrm{sp}}}\right)\right]^{T} \in \mathbb{C}^{N_{\mathrm{sp}} \times 1} .
\end{aligned}
$$

Then the sampled received signal can be written in an equivalent matrix form as

$$
\mathbf{r}_{\mathrm{sp}}=\sum_{k=1}^{K} \sqrt{E_{k}} \overline{\mathbf{s}}_{k} \mathbf{d}_{k}+\mathbf{n}_{\mathrm{sp}} .
$$

Since $n(t)$ is a complex Gaussian random process with zero mean and PSD $N_{0} / 2=\sigma^{2}$ over the whole frequency band, after passing through the ideal LPF with bandwidth $W$, the noise process $n_{\mathrm{lp}}(t)$ becomes a stationary zero mean Gaussian process ([35], Chap. 3 ) with the auto-correlation function

$$
R_{\mathrm{lp}}(\tau)=N_{0} W \operatorname{sinc}(2 W \tau)
$$

where $\operatorname{sinc}(\cdot)$ is the normalized sinc function. Therefore, the sampled additive noise vector $\mathbf{n}_{\mathrm{sp}}$ is a zero mean complex Gaussian random vector with covariance matrix $\mathbf{R}_{\mathrm{sp}}=\left[r_{i j}\right]_{\{i, j\}}, i, j=1,2, \ldots, N_{\mathrm{sp}}$,

$$
r_{i j}=R_{\mathrm{lp}}\left(t_{i}-t_{j}\right), i, j=1,2, \ldots, N_{\mathrm{sp}} .
$$

The capacities with sampling are then similarly obtained as in (8) and (10) with some small modifications; the equivalent matrix $\mathbf{H}_{k}$ needs to be replaced by $\overline{\mathbf{S}}_{k}$, the noise covariance matrix $\sigma^{2} \mathbf{H}$ needs to be replaced by $\mathbf{R}_{\mathrm{sp}}$. Accordingly, let us define $R_{1}^{\mathrm{sp}}, R_{2}^{\mathrm{sp}}, \ldots, R_{K}^{\mathrm{sp}}$ as the maximum number of bits that can be reliably transmitted from user 1 , user $2, \ldots$, user $K$ per block of $N$ symbols assuming sampling is employed at the receiver. The sampling capacity is then characterized by ${ }^{4}$

$$
\sum_{k \in \mathcal{J}} R_{k}^{\mathrm{sp}} \leq \log \operatorname{det}\left(\mathbf{I}_{N_{\mathrm{sp}}}+\sum_{k \in \mathcal{J}} E_{k} \overline{\mathbf{S}}_{k}^{\dagger} \mathbf{R}_{\mathrm{sp}}^{-1} \overline{\mathbf{S}}_{k}\right)
$$

for a Gaussian input signal and

$$
\sum_{k \in \mathcal{J}} R_{k}^{\mathrm{sp}} \leq-\mathbb{E}\left\{\log _{2}\left(f_{\mathbf{r}_{\mathcal{J}}^{\mathrm{sp}}}(\bar{r})\right)\right\}-\log _{2}\left(\operatorname{det}\left(\pi e \mathbf{R}_{\mathrm{sp}}\right)\right)
$$

for a finite constellation input signal, where $\mathbf{r}_{\mathcal{J}}^{\mathrm{sp}} \triangleq$ $\sum_{k \in \mathcal{J}} \sqrt{E_{k}} \overline{\mathbf{S}}_{k} \mathbf{d}_{k}+\mathbf{n}_{\text {sp }}$ is a Gaussian mixture random vector.

\subsection{Numerical characterization}

In this subsection, we numerically characterize the capacity for a two-user uplink WCDMA example. For numerical experiments, we set the parameters which are close to those in a real uplink UMTS system as specified in [1]: time-variant CDMA with orthogonal variable spreading factor (OVSF) codes and Gold sequences, spreading factor $N_{s f}=4$, SRRC chip waveform $p(t)$ with roll-off factor 0.22 , and uniform power allocation $E_{1} / \sigma^{2}=E_{2} / \sigma^{2}=$ SNR. In the simulations, we employ a time-invariant multipath channel with $L=3$ taps, a relative path-amplitude vector $\mathbf{a}=[0,-1.5,-3] \mathrm{dB}$, a relative path-phase vector $\boldsymbol{\theta}=\left[0, \frac{\pi}{3}, \frac{2 \pi}{3}\right]$, and path-delay vector $\boldsymbol{\tau}=\left[0, \frac{T_{c}}{2}, T_{c}\right]$. Thus, the $l$-th element of the path-coefficient vector $\mathbf{g}_{1}$ is given by $a_{l} \cdot \mathrm{e}^{j \theta_{l}} /\|\mathbf{a}\|$ where $a_{l}$ is the $l$-th element of $\mathbf{a}, \theta_{l}$ is the $l$-th element of vector $\boldsymbol{\theta}$, and $\mathbf{g}_{2}=\sqrt{2} \mathbf{g}_{1}$. In addition, we use fixed user delays which are randomly drawn within a symbol time once at the beginning of simulations, i.e., $\lambda_{k} \sim \mathcal{U}\left(0, T_{s}\right)$.

Figure 2 illustrates the capacity of a two-user uplink UMTS system with $N=2$ and $M=1$ for Gaussiandistributed input (from Section 3.1) and 4-QAM (QPSK) input (from Section 3.2) signals. The left-hand side subfigure presents the sum- and individual capacities for Gaussian and 4-QAM input signals. The individual capacities $R_{2}$ are larger than $R_{1}$ since we set $\mathbf{g}_{2}=\sqrt{2} \mathbf{g}_{1}$. The right-hand side sub-figure shows the capacity regions with 4-QAM input for several values of SNR. As expected, the capacity region enlarges with increasing SNR. Moreover, as the SNR tends to infinity, the capacity region converges to the corresponding source entropy outer bound (i.e., 2 bits/symbol individual rates and 4 bits/symbol sum-rate for the two-user channel with 4-QAM input). It is interesting that the maximal individual rates ( 2 bits/symbol) can be achieved simultaneously, i.e., the sum-rate constraint is asymptotically inactive in the high-SNR regime. A deeper analysis on this asymptotic behavior will be given in the next section.

Figure 3 shows the achievable sum-rates for larger block length $(N=32)$ and two codes $(M=2)$ in each I/Q branch. In this figure, both the achievable sum-rates achieved by sufficient statistic (from Sections 3.1-3.2) and by sampling (from Section 3.3) are included. For achievable sum-rates using sampling, the experiments with lower than Nyquist rate $\left(T_{s p}=T_{c}>T_{n y}\right)$ and Nyquist rate $\left(T_{s p}=T_{n y}\right)$ are considered. As expected, the sumcapacity achieved by the sufficient statistic is an upper bound for the sum-rates achieved by systems employing sampling. Moreover, even when the samples are taken with Nyquist rate, there are still gaps between the sum- 

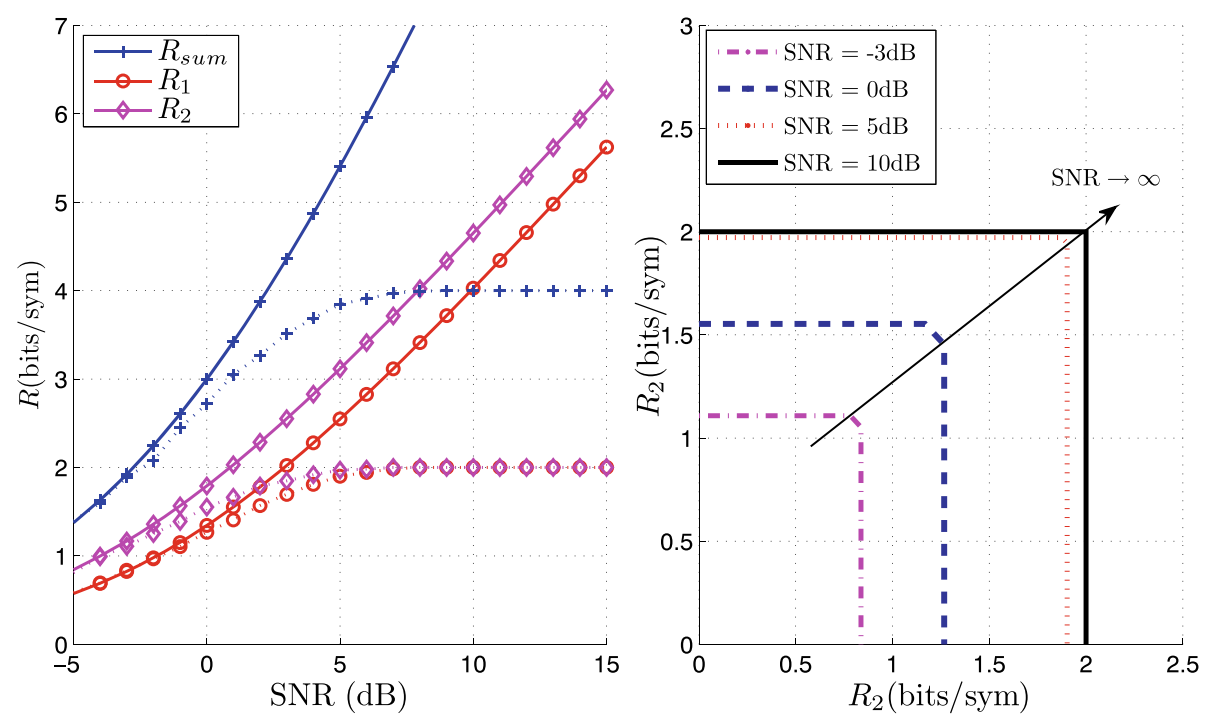

Fig. 2 Capacity curves and capacity regions for a two-user set-up with $N=2$ and $M=1$. On the left-hand side, the solid lines represent the capacities with Gaussian input and the dotted lines represent the capacities with 4-QAM input. All the capacities are normalized by $1 / \mathrm{N}$

rates achieved by sampling $\left(R_{s p}^{\text {Gauss }}\left(T_{s p}=T_{n y}\right)\right.$ and $\left.R_{s p}^{\mathrm{QAM}}\left(T_{s p}=T_{n y}\right)\right)$ and the sum-capacities achieved with matched filtering/sufficient statistic ( $R_{s s}^{\text {Gauss }}$ and $R_{s s}^{\mathrm{QAM}}$ ). These losses are due to the finite time limit of our sampling window as the Nyquist sampling theorem states that a infinite sample sequence is required to be able to perfectly recover a finite energy and band-limited signal ([26], Theorem 8.4.3). Fortunately, by extending the sampling window by only two symbol durations on each side (for $R_{s p+}^{\mathrm{Gauss}}$ and $R_{s p+}^{\mathrm{QAM}}$ ), these losses can be significantly reduced.

\section{Asymptotic analysis}

Recalling (7) with $\mathcal{J}=\{1, \ldots, K\}$, we have $\sum_{k=1}^{K} R_{k} \leq$ $I(\mathbf{d} ; \mathbf{z}) \leq \sum_{k=1}^{K} H\left(\mathbf{d}_{k}\right)$, i.e., the sum-capacity is upper bounded by the sum of the sources entropies. However, the results in Fig. 2 show that when SNR $\rightarrow \infty$, the individual capacities can be simultaneously achieved, i.e., the sum-capacity approaches the sum of the individual source entropies. In this section, we provide a deeper analysis on this observation by characterizing the asymptotic behavior of the sum-capacity. For convenience, we begin with a simple MAC model then extend the result to the uplink WCDMA system in the following.

\subsection{Simple $K$-user MAC model}

Firstly, we start from the asymptotic sum-capacity of a simple $K$-user MAC, where each user transmits only one data stream. This setup corresponds to our uplink WCDMA system with $M=1$ and $N=1$ in a frequencynon-selective channel. The results are mainly based on the following lemma.
Lemma 1. Consider the received signal model of a $K$ user $M A C$

$$
y(t)=\sum_{k=1}^{K} d_{k} s_{k}(t)+n(t)
$$

where $d_{1}, \ldots, d_{K}$ are the unit power transmitted symbols, which are independent and transmitted using $K$ normalized signature waveforms $s_{1}(t), \ldots, s_{K}(t)$ and $n(t)$ denotes the Gaussian noise process with PSD $\frac{1}{S N R}$. When SNR $\rightarrow \infty$, the asymptotic sum-capacity of channel (15), $C_{\text {sum }}^{\text {as }}=\sum_{k=1}^{K} H\left(d_{k}\right)$, is achieved if the vector space $\mathcal{S}_{K}=\operatorname{span}\left\{s_{1}(t), \ldots, s_{K}(t)\right\}$ has the dimension K.

The proof of Lemma 1 is given in Appendix 2. The idea for the proof is that we first show that the received signal passing through a bank of matched filters, which are matched to the signature waveforms, yields a sufficient statistic for decoding $\mathbf{d}=\left[d_{1}, \cdots, d_{K}\right]$ based on $y(t)$. Then, we show that $\mathbf{d}$ can be uniquely decoded, i.e., the decoder is able to decode the messages correctly from this sufficient statistic when SNR $\rightarrow \infty$ if $\operatorname{dim}\left(\mathcal{S}_{K}\right)=K$. Based on the uniquely decodable property, we then prove that the asymptotic sum-capacity $C_{\text {sum }}^{\text {as }}$ approaches the sum of source entropies if the signature waveforms are linearly independent of each other, i.e., $\operatorname{dim}\left(\mathcal{S}_{K}\right)=K$.

\subsection{Uplink WCDMA system}

Next, we extend the results from the above simple $K$ user MAC to the asymptotic sum-capacity of the uplink 


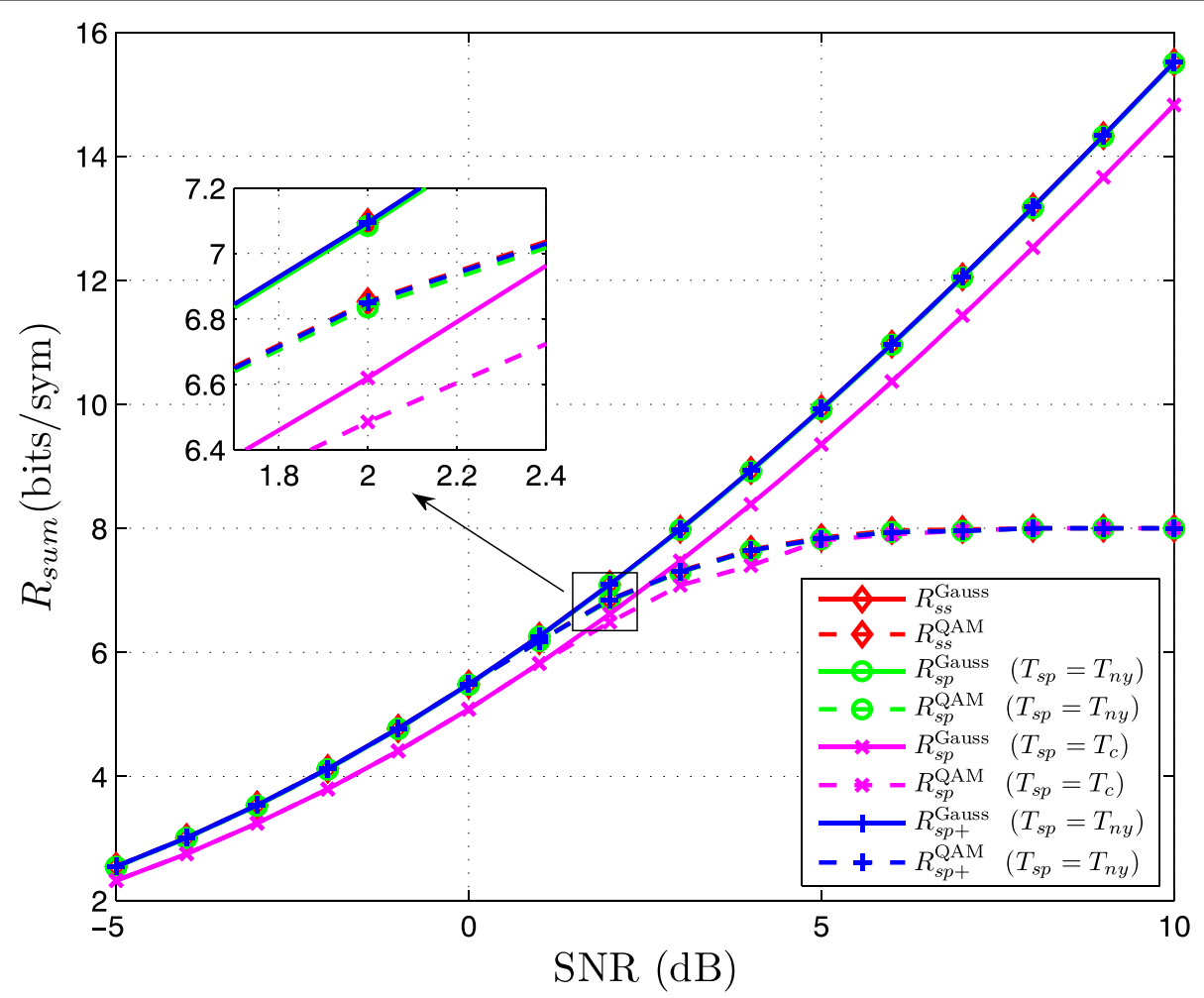

Fig. 3 Achievable sum-rates for different inputs and receiver structures with $N=32$ and $M=2 . R_{s s}^{\text {Gauss }}$ and $R_{s s}^{\text {QAM }}$ denote the sum-rates achieved by the sufficient statistic with Gaussian input and 4-QAM input, respectively. $R_{S P}^{\mathrm{Gauss}}$ and $R_{S p}^{\mathrm{QAM}}$ denote the sum-rates achieved by sampling with the sampling window equal to the block length $t_{n} \in\left[\begin{array}{ll}0 & N T_{s}\end{array}\right]$. $R_{s p+}^{\text {Gauss }}$ and $R_{s p+}^{\mathrm{QAM}}$ denote the sum-rates achieved by sampling with the sampling window extended by two symbol durations on each side, i.e., $t_{n} \in\left[-2 T_{s}(N+2) T_{s}\right]$. All the sum-rates are normalized by $1 / N$

WCDMA system. Let us recall the transmitted signal from user $k$ of the uplink WCDMA system in (1), and take into account all $K$ users. The transmitted signal can be considered as one of equivalent $K N M$-user MACs in (15) using $K N M$ signature waveforms $s_{k i}^{m}\left(t-\lambda_{k}\right), k=1, \ldots, K, i=$ $1, \ldots, N$, and $m=1, \ldots, M$. The following propositions, which can be derived from Lemma 1, specify the asymptotic sum-capacity of the uplink WCDMA system in different channel environments considering frequencynon-selective $(L=1)$ and frequency-selective $(L \geq 2)$ channels.

Proposition 1. The asymptotic sum-capacity of the frequency-non-selective uplink WCDMA channel as described in (2) with $L=1$ is $C_{\text {sum,nsec }}^{\text {as }}=\sum_{k=1}^{K} H\left(\mathbf{d}_{k}\right)$ if the dimension of the signature waveforms space $\mathcal{S}_{T}=\operatorname{span}\left\{s_{11}^{1}\left(t-\lambda_{1}\right), \ldots, s_{K N}^{M}\left(t-\lambda_{K}\right)\right\}$ is $K N M$.

The proof for Proposition 1 is given in Appendix 3. The intuition behind Proposition 1 can be expressed as: $K$ users transmit $K N M$ symbols and the receiver performs matched filtering with KNM fingers. Although the uplink WCDMA multiuser channel implies $K$-user SISO MACs, the matching process virtually converts this to an equivalent $K N M \times K N M$ MIMO channel. Thus, by appropriately choosing the signature waveforms and matched fingers, which yield a full-rank equivalent channel matrix $\mathbf{H}$, the transmitted symbols, $\mathbf{d}_{1}, \ldots, \mathbf{d}_{K}$, can be perfectly (i.e., error-free) recovered from $\mathbf{z}$ as SNR goes to infinity. In other words, a $K$-user uplink WCDMA channel can asymptotically achieve the capacity of $K N M$ parallel channels as long as $\operatorname{dim}\left(\mathcal{S}_{T}\right)=K N M$.

Proposition 2. The asymptotic sum-capacity of the frequency-selective uplink WCDMA channel as described in (2) is $C_{\text {sum }, \text { sec }}^{a s}=\sum_{k=1}^{K} H\left(\mathbf{d}_{k}\right)$ if $\operatorname{dim}(\overline{\mathcal{S}})=K N M$, where $\overline{\mathcal{S}}=\operatorname{span}\left\{\bar{s}_{11}^{1}(t), \cdots, \bar{s}_{K N}^{M}(t)\right\}$ is the vector space spanned by the effective signature waveform $\bar{s}_{k i}^{m}(t)=\sum_{l=1}^{L} g_{k l} s_{k i}^{m}$ $\left(t-\lambda_{k}-\tau_{k l}\right)$.

The proof for Proposition 2 is given in Appendix 4 . Unlike the frequency-non-selective channel case, the sufficient condition for achieving the asymptotic sumcapacity in a frequency-selective channel case is based on the effective signature waveforms, which include the impact of the channel gains $\left\{\mathbf{g}_{k}\right\}_{k}$ and delays $\left\{\tau_{k l}\right\}_{k, l}$. This implies that the multi-path channel may help the 
equivalent channel matrix $\mathbf{H}$ to achieve full-rank. For instance, if $\operatorname{dim}\left(\mathcal{S}_{T}\right)<K N M, \mathbf{H}$ is obviously singular when $L=1$, while $\mathbf{H}$ can be still invertible when $L>1$ since $\operatorname{dim}(\overline{\mathcal{S}})$ is possible to be equal to $K N M$ according to the channel selectivity and the potential offset in the multi-path environment ${ }^{5}$. This is particularly helpful in an overloaded CDMA system [8, 21], where the number of users exceed the spreading factor.

\subsection{Necessary condition}

Propositions 1 and 2 state sufficient conditions that the transmitted messages can be uniquely decoded when SNR $\rightarrow \infty$, which holds for all kinds of input signals including both finite and infinite constellation signals. However, the conditions in Propositions 1 and 2 can be relaxed in certain scenarios with finite constellation inputs. In this subsection, we first consider a simple example where such conditions can be relaxed. The necessary condition for the unique decoding with finite constellation input is then studied in the following.

For instance, let us consider an example with two different set-ups of channel (15) with $K=2$ and binary transmitted signals $\mathbf{d}_{a}=\left[\begin{array}{ll}d_{a 1} & d_{a 2}\end{array}\right]^{T}$ and $\mathbf{d}_{b}=\left[\begin{array}{ll}d_{b 1} & d_{b 2}\end{array}\right]^{T}$, i.e.,

$$
\begin{aligned}
& y_{a}(t)=d_{a 1} s_{1}(t)+d_{a 2} s_{2}(t)+n_{a}(t), \\
& y_{b}(t)=d_{b 1} s_{1}(t)+d_{b 2} s_{2}(t)+n_{b}(t),
\end{aligned}
$$

where $n_{a}(t)$ and $n_{b}(t)$ denote additive Gaussian noise processes. We assume that the same signature waveform space $\mathcal{S}_{2}:=\operatorname{span}\left\{s_{1}(t), s_{2}(t)\right\}$ is used in both set-ups. However, the transmitted symbols are uniformly and randomly picked up from different input constellation sets: $d_{a 1}, d_{a 2} \in\{0,1\}$ and $d_{b 1} \in\{-1 / \sqrt{2}, 1 / \sqrt{2}\}, d_{b 2} \in\{0,1\}$. The corresponding sufficient statistic models are then given by

$$
\begin{aligned}
& Y_{a}=d_{a 1}+d_{a 1}+N_{a}, \\
& Y_{b}=d_{b 1}+d_{b 2}+N_{b},
\end{aligned}
$$

where $Y_{a}$ and $Y_{b}$ denote the sufficient statistics and $N_{a}$ and $N_{b}$ are the equivalent noises. We can see that when the noise power becomes zero (or SNR $\rightarrow \infty$ ), $\mathbf{d}_{a}$ (and so $d_{a 1}$ and $\left.d_{a 2}\right)$ ) cannot be uniquely decoded from $Y_{a}$ since the conditional entropy $H\left(d_{a 1}, d_{a 2} \mid Y_{a}\right)=0.5>0$ when SNR $\rightarrow \infty$. However, $\left(d_{b 1}\right.$ and $\left.d_{b 2}\right)$ can be uniquely decoded from $Y_{b}$ since $H\left(d_{b 1}, d_{b 2} \mid Y_{b}\right)=0$ when SNR $\rightarrow$ $\infty$ even though $\operatorname{dim}\left(\mathcal{S}_{2}\right)=1<2$. It shows that the con$\operatorname{dition} \operatorname{dim}\left(\mathcal{S}_{K}\right)=K$ can be relaxed for certain signal constellation structures. Therefore, it is expected that necessary conditions for achieving the unique decoding with finite constellation input have to take both the signature waveforms and the structure of the signal constellation into account.
Let us assume that $\mathbf{d} \in \mathcal{M}^{K N M}$, where $\mathcal{M}$ is a set of constellation points and is finite. In order to derive the sufficient condition for the unique decoding, we refer the equivalent channel in (C.2) in Appendix 3

$$
\mathbf{z}=\text { HEd }+\mathbf{n} .
$$

When SNR $\rightarrow \infty$, the transmitted vector $\mathbf{d}$ can be uniquely decoded from $\mathbf{z}$ if and only if the mapping

$$
\begin{aligned}
f: \mathcal{M}^{K N M} & \mapsto \mathcal{C}^{K N M} \\
\mathbf{d} & \mapsto \text { HEd }
\end{aligned}
$$

is an one-to-one mapping. In particular, for any pair of $\mathbf{d}^{i}, \mathbf{d}^{j} \in \mathcal{M}^{K N M}$ and $\mathbf{d}^{i} \neq \mathbf{d}^{j}$, the condition $\mathbf{H E d ^ { i }} \neq \mathbf{H E d} \mathbf{d}^{j}$ is needed for the unique decoding. Therefore, by defining $\mathbf{v}_{i j}=\mathbf{d}^{i}-\mathbf{d}^{j}$, the condition for the unique decoding becomes

$$
\mathbf{H E v}_{i j} \neq 0, \forall i \neq j .
$$

In other words, the necessary condition for the unique decoding is that any vector $\mathbf{v}_{i j}$ with $i \neq j$ is not in the null space of matrix HE. This necessary condition includes the impact of signal constellation reflected via $\mathbf{v}_{i j}$.

Remark 2. This result is consistent with the sufficient conditions in Propositions 1 and 2. Indeed, when the (effective) signature waveform space has dimension KNM and $\mathbf{H}$ is invertible, the null space of $\mathbf{H E}$ is empty. Thus, the condition in (17) holds for any set of vector $\mathbf{v}_{i j}$, and the unique decoding is achieved for any kind of input signal.

\section{Conclusions}

This paper studies the capacity limit of the uplink WCDMA system whose set-up has been chosen to be close to real CDMA cellular networks. We present a theoretical framework, which can be used to evaluate how close the maximal performance of a practical system design is to the theoretical fundamental limit. To this end, sufficient statistics for decoding the transmitted messages were derived using a bank of matched filters, each of which is matched to the signature waveforms. An equivalent discrete-time channel model based on the derived sufficient statistics was provided which can be used to analyze the capacity of the system. The capacity regions for finite constellation input and Gaussian-distributed input signals have been both analytically and numerically characterized. The comparison with the sampling capacity showed that sampling within the transmission time window might cause a capacity loss even if the sampling was performed at Nyquist rate. Fortunately, this loss could be significantly diminished by extending the sampling window by only two symbol durations. Moreover, the asymptotic analysis shows that for proper choices of the (effective) signature waveforms, a $K$-user uplink 
WCDMA channel can be decoupled so that each user achieves a point-to-point channel capacity when SNR goes to infinity. The presented framework and results provide valuable insights for the design and further development of not only WCDMA but also other wireless standard networks.

\section{Appendices} Appendix 1 Derivation of equivalent noise statistic Since $n(t)$ is a zero mean complex Gaussian random process, the equivalent noises after a bank of linear filters (matched filters), $n_{k i}^{m l}=\int_{-\infty}^{\infty} n(t) s_{k i}^{m}\left(t-\lambda_{k}-\tau_{k l}\right)^{*} d t$, $\forall k, i, m, l$, are zero mean joint Gaussian random variables ([28], Chap. 8) with the correlation coefficient given by

$$
\begin{aligned}
& \mathbb{E}\left\{n_{k i}^{m l} n_{k^{\prime} i^{\prime}}^{m^{\prime} l^{*}}\right\} \\
& =\mathbb{E}\left\{\int_{-\infty}^{\infty} n(t) s_{k i}^{m}\left(t-\lambda_{k}-\tau_{k l}\right)^{*} d t \int_{-\infty}^{\infty} n\left(t^{\prime}\right)^{*} s_{k^{\prime} i^{\prime}}^{m^{\prime}}\left(t^{\prime}-\lambda_{k^{\prime}}-\tau_{k^{\prime} l^{\prime}}\right) d t^{\prime}\right\} \\
& =\int_{-\infty}^{\infty} \int_{-\infty}^{\infty} \mathbb{E}\left\{n(t) n\left(t^{\prime}\right)^{*}\right\} s_{k i}^{m}\left(t-\lambda_{k}-\tau_{k l}\right)^{*} s_{k^{\prime} i^{\prime}}^{m^{\prime}}\left(t^{\prime}-\lambda_{k^{\prime}}-\tau_{k^{\prime} l^{\prime}}\right) d t d t^{\prime},
\end{aligned}
$$

s where $\mathbb{E}\left\{n(t) n\left(t^{\prime}\right)^{*}\right\}=\sigma^{2} \delta\left(t-t^{\prime}\right)$. Thus, we have

$$
\begin{aligned}
\mathbb{E} & \left\{n_{k i}^{m l} n_{k^{\prime} i^{\prime}}^{m^{\prime} l^{\prime}}\right\} \\
& =\int_{-\infty}^{\infty} \int_{-\infty}^{\infty} \sigma^{2} \delta\left(t-t^{\prime}\right) s_{k i}^{m}\left(t-\lambda_{k}-\tau_{k l}\right)^{*} s_{k^{\prime} i^{\prime}}^{m^{\prime}}\left(t^{\prime}-\lambda_{k^{\prime}}-\tau_{k^{\prime} l^{\prime}}\right) d t d t^{\prime} \\
& =\sigma^{2} \int_{-\infty}^{\infty} s_{k i}^{m}\left(t-\lambda_{k}-\tau_{k l}\right)^{*}\left[\int_{-\infty}^{\infty} \delta\left(t-t^{\prime}\right) s_{k^{\prime} i^{\prime}}^{m^{\prime}}\left(t^{\prime}-\lambda_{k^{\prime}}-\tau_{k^{\prime} l^{\prime}}\right) d t^{\prime}\right] d t \\
& =\sigma^{2} \int_{-\infty}^{\infty} s_{k i}^{m}\left(t-\lambda_{k}-\tau_{k l}\right)^{*} s_{k^{\prime} i^{\prime}}^{m^{\prime}}\left(t-\lambda_{k^{\prime}}-\tau_{k^{\prime} l^{\prime}}\right) d t \\
& =\sigma^{2} \rho_{(k i m l)}^{\left(k^{\prime} i^{\prime} m^{\prime} l^{\prime}\right)} .
\end{aligned}
$$

Accordingly, the equivalent noises $n_{k i}^{m}=\sum_{l=1}^{L} g_{k l}{ }^{*} n_{k i}^{m l}$, $\forall k, i, m$ are zero mean joint Gaussian random variables with correlation coefficient

$$
\begin{aligned}
\mathbb{E}\left\{n_{k i}^{m} n_{k^{\prime} i^{\prime}}^{m^{\prime}}\right\} & =\sum_{l=1}^{L} \sum_{l^{\prime}=1}^{L} g_{k l^{*}} g_{k^{\prime} l^{\prime}} \mathbb{E}\left\{n_{k i}^{m l} n_{k^{\prime} i^{\prime}}^{m^{\prime} l^{\prime *}}\right\} \\
& =\sigma^{2} \sum_{l=1}^{L} \sum_{l^{\prime}=1}^{L} g_{k l^{*}} g_{k^{\prime} l^{\prime}} \rho_{(k i m l)}^{\left(k^{\prime} i^{\prime} m^{\prime} l^{\prime}\right)} .
\end{aligned}
$$

Moreover, we have the $(a, b)$ th element of $\mathbf{H}$ expressed as

$$
\mathbf{H}[a, b]=\sum_{l=1}^{L} \sum_{l^{\prime}=1}^{L} g_{k l}{ }^{*} g_{k^{\prime} l^{\prime}} \rho_{(k i m l)}^{\left(k^{\prime} i^{\prime} m^{\prime} l^{\prime}\right)},
$$

where the indices are given by

$$
\begin{aligned}
& a=(k-1) N M+(i-1) M+m, \\
& b=\left(k^{\prime}-1\right) N M+\left(i^{\prime}-1\right) M+m^{\prime} .
\end{aligned}
$$

As a result, $\mathbf{n}$ is a complex Gaussian random vector with zero mean and covariance matrix $\sigma^{2} \mathbf{H}$.

\section{Appendix 2 Proof of Lemma 1}

The proof of Lemma 1 consists of two parts:

- Part 1: We first show that the received signal passed through a bank of matched filters, which match to the signature waveforms, yields a sufficient statistic for decoding $\mathbf{d}=\left[d_{1}, \cdots, d_{K}\right]^{T}$ based on $y(t)$. Moreover, when SNR $\rightarrow \infty$, d can be uniquely decoded if $\operatorname{dim}\left(\mathcal{S}_{K}\right)=K$.

- Part 2: Based on the uniquely decodable property, we then derive the asymptotic sum-capacity.

Part 1: Part 1 is a result of the following claim

Claim. Let the received signal $y(t)$ in (15) passed through a bank of matched filters, where $y(t)$ is matched with each signature waveform $s_{k}(t)$, i.e.,

$$
y_{k}=\left\langle y(t), s_{k}(t)\right\rangle=\int_{-\infty}^{\infty} y(t) s_{k}^{*}(t) d t, \quad k=1 \cdots K .
$$

Then $\mathbf{y}=\left[y_{1}, \cdots, y_{K}\right]^{T}$ is a sufficient statistic for decoding $\mathbf{d}$ based on $y(t)$. Moreover, if the vector space $\mathcal{S}_{K}=$ $\operatorname{span}\left\{s_{1}(t), \cdots, s_{K}(t)\right\}$ has a dimension of $K, \mathbf{d}$ can be uniquely decoded from the sufficient statistic $y$ as SNR $\rightarrow$ $\infty$.

Proof. Following similar steps as in Section 2.2, it can be shown that $\mathbf{y}$ is a sufficient statistic for decoding $\mathbf{d}$ based on $y(t)$.

It remains to show that $\mathbf{d}$ can be uniquely decoded from y when SNR $\rightarrow \infty$. Let us denote $\mathbf{R}_{s}$ as the correlation matrix of the signature waveforms $\left\{s_{k}(t)\right\}_{k}$, where $\mathbf{R}_{s}[i, j]=\left\langle s_{i}(t), s_{j}(t)\right\rangle$. Therewith, we have the equivalent matrix expression

$$
\mathbf{y}=\mathbf{R}_{s} \mathbf{d}+\tilde{\mathbf{n}},
$$

where $\widetilde{\mathbf{n}}$ is the equivalent noise vector.

Since $\operatorname{dim}\left(\mathcal{S}_{K}\right)=K$, we can rewrite $\left\{s_{1}(t), \cdots, s_{K}(t)\right\}$ as

$$
\left[\begin{array}{c}
s_{1}(t) \\
\vdots \\
s_{K}(t)
\end{array}\right]=\mathbf{A}\left[\begin{array}{c}
e_{1}(t) \\
\vdots \\
e_{K}(t)
\end{array}\right]
$$

where $\left\{e_{1}(t), \cdots, e_{K}(t)\right\}$ is an orthonormal basis of $\mathcal{S}_{K}$ and $\mathrm{A}$ is a $K \times K$ full rank matrix.

Consider the correlation matrix $\mathbf{R}_{e}$ where

$$
\mathbf{R}_{e}[i, j]=\left\langle e_{i}(t), e_{j}(t)\right\rangle .
$$

Then, we have $\mathbf{R}_{e}=\mathbf{I}_{K}$ since $\left\{e_{1}(t), \cdots, e_{K}(t)\right\}$ is an orthonormal basic. Moreover,

$$
\mathbf{R}_{s}=\mathbf{A R}_{e} \mathbf{A}^{\dagger}=\mathbf{A A}^{\dagger} .
$$

Thus,

$$
\operatorname{rank}\left(\mathbf{R}_{s}\right)=\operatorname{rank}\left(\mathbf{A A}^{\dagger}\right)=\operatorname{rank}(\mathbf{A})=K .
$$


Therefore, when $\operatorname{dim}\left(\mathcal{S}_{K}\right)=K, \mathbf{R}_{s}$ is invertible and $\mathbf{d}$ can be uniquely decoded from $\mathbf{y}$ when SNR $\rightarrow \infty$ since $\lim _{\mathrm{SNR} \rightarrow \infty} \mathbf{R}_{s}^{-1} \mathbf{y}=\mathbf{d}$.

Part 2: We next derive the asymptotic sum-capacity based on the sufficient statistic from Part 1.

The sum-capacity of the channel (15) is given by

$$
C_{\text {sum }}=I(\mathbf{d} ; y(t)) \text {. }
$$

From Part 1, we know that $\mathbf{y}$ is a sufficient statistic for decoding $\mathbf{d}$ based on $y(t)$. Thus,

$$
I(\mathbf{d} ; y(t))=I(\mathbf{d} ; \mathbf{y})
$$

where

$$
I(\mathbf{d} ; \mathbf{y})=H(\mathbf{d})-H(\mathbf{d} \mid \mathbf{y}) .
$$

When $\operatorname{dim}\left(\mathcal{S}_{K}\right)=K$, following from Part $1, \mathbf{R}_{s}$ is invertible and $\lim _{S N R \rightarrow \infty} \mathbf{R}_{s}^{-1} \mathbf{y}=\mathbf{d}$. Therefore,

$$
\lim _{\mathrm{SNR} \rightarrow \infty} H(\mathbf{d} \mid \mathbf{y})=H\left(\mathbf{R}_{s}^{-1} \mathbf{y} \mid \mathbf{y}\right)=0
$$

Let us define the asymptotic sum-capacity as $C_{\text {sum }}^{\text {as }}=$ $\lim _{\mathrm{SNR} \rightarrow \infty} C_{\text {sum }}$, combining (B.1)-(B.4), we have

$$
C_{\mathrm{sum}}^{\mathrm{as}}=H(\mathbf{d})=\sum_{k=1}^{K} H\left(d_{k}\right) .
$$

This completes the proof for Lemma 1.

\section{Appendix 3 Proof of Proposition 1}

Proposition 1 is proved in three steps:

- Step 1: We first re-formulate (5) by an equivalent input-output model, in which $\mathbf{H}$ is decomposed into a multiplication of multiple matrices including a matrix that depends only on the signature waveform correlation coefficients. To this end, we rewrite the equivalent channel $\mathbf{H}$ as follows:

$$
\begin{aligned}
\mathbf{H}= & {\left[\mathbf{H}_{1}, \ldots, \mathbf{H}_{K}\right] } \\
= & {\left[\begin{array}{cccc}
\mathbf{G}_{1}^{\dagger} \mathbf{R}_{1}^{1} \mathbf{G}_{1} & \mathbf{G}_{1}^{\dagger} \mathbf{R}_{1}^{2} \mathbf{G}_{2} & \cdots & \mathbf{G}_{1}^{\dagger} \mathbf{R}_{1}^{K} \mathbf{G}_{K} \\
\mathbf{G}_{2}^{\dagger} \mathbf{R}_{2}^{1} \mathbf{G}_{1} & \mathbf{G}_{2}^{\dagger} \mathbf{R}_{2}^{2} \mathbf{G}_{2} & \cdots & \mathbf{G}_{2}^{\dagger} \mathbf{R}_{2}^{K} \mathbf{G}_{K} \\
\vdots & \vdots & \vdots & \vdots \\
\mathbf{G}_{K}^{\dagger} \mathbf{R}_{K}^{1} \mathbf{G}_{1} & \mathbf{G}_{K}^{\dagger} \mathbf{R}_{K}^{2} \mathbf{G}_{2} & \cdots & \mathbf{G}_{K}^{\dagger} \mathbf{R}_{K}^{K} \mathbf{G}_{K}
\end{array}\right] . }
\end{aligned}
$$

Therefore, the equivalent channel $\mathbf{H}$ can be expressed as

$$
\mathbf{H}=\mathbf{G}^{\dagger} \mathbf{R G}
$$

where

$$
\mathbf{G}=\operatorname{blkdiag}\left(\mathbf{G}_{1}, \ldots, \mathbf{G}_{K}\right) \in \mathbb{C}^{K N M L \times K N M},
$$

and

$$
\mathbf{R}=\left[\begin{array}{cccc}
\mathbf{R}_{1}^{1} & \mathbf{R}_{1}^{2} & \cdots & \mathbf{R}_{1}^{K} \\
\mathbf{R}_{2}^{1} & \mathbf{R}_{2}^{2} & \cdots & \mathbf{R}_{2}^{K} \\
\vdots & \vdots & \vdots & \vdots \\
\mathbf{R}_{K}^{1} & \mathbf{R}_{K}^{2} & \cdots & \mathbf{R}_{K}^{K}
\end{array}\right] \in \mathbb{C}^{K N M L \times K N M L}
$$

Thus, we have the equivalent input-output model as

$$
\mathbf{z}=\mathbf{H E d}+\mathbf{n},
$$

where

$$
\mathbf{E}=\operatorname{diag}(\underbrace{\sqrt{E_{1}}, \ldots, \sqrt{E_{1}}}_{N M \text { elements }}, \ldots, \underbrace{\sqrt{E_{K}}, \ldots, \sqrt{E_{K}}}_{N M \text { elements }}) .
$$

- Step 2: We show that $\mathbf{H}$ is invertible when $L=1$ and $\operatorname{dim}\left(\mathcal{S}_{T}\right)=K N M$

Following the same arguments as for the proof of Lemma 1, we have $\mathbf{R}$ as full rank since $\operatorname{dim}\left(\mathcal{S}_{T}\right)=K N M$. Since $\mathbf{H}=\mathbf{G}^{\dagger} \mathbf{R G}$, where $\mathbf{G}, \mathbf{R}$ are the square matrices with full rank, it follows that $\mathbf{H}$ is invertible.

- Step 3: Lastly, we can conclude on the asymptotic capacity of the channel (5). Since $\mathbf{z}$ in (C.2) is a sufficient statistic for decoding $\mathbf{d}$ based on $y(t)$ (from Section 2.2) and $\mathbf{H}$ is invertible, similarly to the proof in Part 2 of Lemma 1, it follows that

$$
C_{\text {sum,nsec }}^{\text {as }}=H(\mathbf{d})=\sum_{k=1}^{K} H\left(\mathbf{d}_{k}\right) .
$$

This completes the proof for Proposition 1.

\section{Appendix 4 Proof of Proposition 2}

Let us define $\bar{\rho}_{k i m}^{k^{\prime} i^{\prime} m^{\prime}}$ as the inner product between $\bar{s}_{k i}^{m}(t)$ and $\bar{s}_{k^{\prime} i^{\prime}}^{m^{\prime}}(t)$, i.e.,

$$
\begin{aligned}
\bar{\rho}_{k i m}^{k^{\prime} i^{\prime} m^{\prime}} & =\left\langle\bar{s}_{k i}^{m}(t), \bar{s}_{k^{\prime} i^{\prime}}^{m^{\prime}}(t)\right\rangle \\
& =\sum_{l=1}^{L} \sum_{l^{\prime}=1}^{L} g_{k l}^{*}\left\langle s_{k i}^{m}\left(t-\lambda_{k}-\tau_{k l}\right), s_{k^{\prime} i^{\prime}}^{m^{\prime}}\left(t-\lambda_{k}-\tau_{k^{\prime} l^{\prime}}\right)\right\rangle g_{k^{\prime} l^{\prime}} \\
& =\sum_{l=1}^{L} \sum_{l^{\prime}=1}^{L} g_{k l}^{*} \rho_{k i m l}^{k^{\prime} i^{\prime} m^{\prime} l} g_{k^{\prime} l^{\prime}} .
\end{aligned}
$$

Define $\mathbf{R}_{\bar{s}}$ as the correlation matrix of $\overline{\mathcal{S}}$, where

$$
\mathbf{R}_{\bar{s}}[a, b]=\bar{\rho}_{k i m}^{k^{\prime} i^{\prime} m^{\prime}},
$$

and the coefficient indices are given by

$$
\begin{aligned}
& a=(k-1) N M+(i-1) M+m, \\
& b=\left(k^{\prime}-1\right) N M+\left(i^{\prime}-1\right) M+m^{\prime} .
\end{aligned}
$$

Since $\mathbf{R}_{\bar{S}}$ is the correlation matrix of $\overline{\mathcal{S}}$, following similar steps as for the proof in Part 1 of Lemma 1, we arrive at $\operatorname{rank}\left(R_{\bar{S}}\right)=K N M$ if $\operatorname{dim}(\overline{\mathcal{S}})=K N M$. 
Moreover, combining (A.1) with (D.1) and (D.2), we have

$$
\mathbf{H}=\mathbf{R}_{\bar{s}} .
$$

Thus, $\operatorname{rank}(\mathbf{H})=K N M$ and $\mathbf{H}$ is invertible.

Finally, similar to the proof in Part 2 of Lemma 1, given $\mathbf{H}$ is invertible, the asymptotic sum-capacity is given by

$$
C_{\text {sum }, \text { sec }}^{\text {as }}=H(\mathbf{d})=\sum_{k=1}^{K} H\left(\mathbf{d}_{k}\right) .
$$

This completes the proof for Proposition 2.

\section{Endnotes}

${ }^{1}$ In theory, a band-limited signal requires infinite time to transmit. However, in practical WCDMA systems, the chip waveforms with fast decaying sidelobes (e.g., root raised cosine (RRC) and squared-root raised cosine (SRRC) pulses) are used and truncated by the length of several chip intervals.

${ }^{2}$ It is worth noting that even though the channel impulse response is assumed to be time-invariant as similar to [12, 24], the Toeplitz structure of the equivalent channel matrix is not maintained because of the variation of the spreading sequences over symbols in a time-variant CDMA system.

${ }^{3}$ In [27], [Proposition 3.2], the hypotheses are equiprobable and the optimal decision is based on maximum of $\Phi(\mathbf{d})$ (ML criterion). In general, the optimal decision is based on MAP criterion, which includes $\log (p(\mathbf{d}))$ into $\mu(t ; \mathbf{d})$. However, this additional term is independent of $r(t)$. Therefore, we do not have to include it in the sufficient statistic.

${ }^{4}$ Similarly to the capacity achieved by sufficient statistic, the quantities in the right-hand sides of (13) and (14) describe the number of bits that can be reliably transmitted per block of $N$ symbols. One can express the capacity in bit/second by normalizing with $1 / T$, $T=N T_{s}$, or in bit/second/Hertz by normalizing with $1 / T W$.

${ }^{5}$ When $L>1$, the channel gain matrix $\mathbf{G}$ is not a square matrix anymore. The invertible property of $\mathbf{H}$ does not depend only on rank of the correlation matrix $\mathbf{R}$ but also on $\mathbf{G}$.

\section{Competing interests}

The authors declare that they have no competing interests.

\section{Author details}

${ }^{1}$ School of Electrical Engineering, KTH Royal Institute of Technology, Stockholm, Sweden. ${ }^{2}$ Department of Electronics Engineering, Korea Polytechnic University, 15073237 Sangidaehak-ro, Gyeonggi-do, Korea. ${ }^{3}$ R\&D Center, Huawei Technologies Sweden AB, Stockholm, Sweden.

Received: 23 June 2015 Accepted: 10 November 2015

Published online: 02 December 2015

\section{References}

1. 3GPP, 3GPP Specification series: 25-series 3GPP TSG RAN (Release 11) (2012). [Online]: http://www.3gpp.org/DynaReport/25-series.htm

2. H Holma, A Toskala, WCDMA for UMTS. (John Wiley \& Sons, New York, USA, 2004)

3. J Hämäläinen, K Pajukoski, E Tiirola, R Wichman, J Ylitalo, On the performance of multiuser MIMO in UTRA FDD uplink. EURASIP J. Wirel. Commun. Netw. 2004(2), 297-308 (2004)

4. JW Park, B Razavi, in Paper presented at the 2014 IEEE International Solid-State Circuits Conference (ISSCC). 20.8 A 20mW GSM/WCDMA receiver with RF channel selection (San Francisco, CA, USA, 2014), pp. 356-357

5. MT Nguyen, C Jabbour, M Homayouni, D Duperrayy, P Triairey, VT Nguyen, in Paper presented at the 12th IEEE New Circuits and Systems Conference (NEWCAS). A flexible direct delta-sigma receiver for GSM/WCDMA/LTE (IEEE Canada, 2014), pp. 357-360

6. M Rupf, J Massey, Optimum sequence multisets for synchronous code-division multiple-access channels. IEEE Trans. Inf. Theory. 40(4), 1261-1266 (1994)

7. P Viswanath, $V$ Anantharam, Optimal sequences and sum capacity of synchronous CDMA systems. IEEE Trans. Inf. Theory. 45(6), 1984-1991 (1999)

8. O Mashayekhi, F Marvasti, Uniquely decodable codes with fast decoder for overloaded synchronous CDMA systems. IEEE Trans. Commun. 60(11), 3145-3149 (2012)

9. SVerdú, S Shamai, Spectral efficiency of CDMA with random spreading. IEEE Trans. Inf. Theory. 45(2), 622-640 (1999)

10. D Tse, S Hanly, Linear multiuser receivers: effective interference, effective bandwidth and user capacity. IEEE Trans. Inf. Theory. 45(2), 641-657 (1999)

11. P Pan, Y Zhang, Y Sun, LL Yang, On the asymptotic spectral efficiency of uplink MIMO CDMA systems over Rayleigh fading channels with arbitrary spatial correlation. IEEE Trans. Veh. Technol. 62(2), 679-691 (2013)

12. S Verdú, The capacity region of the symbol-asynchronous Gaussian multiple-access channel. IEEE Trans. Inf. Theory. 35(4), 733-751 (1989)

13. S Ulukus, R Yates, User capacity of asynchronous CDMA systems with matched filter receivers and optimum signature sequences. IEEE Trans. Inf. Theory. 50(5), 903-909 (2004)

14. J Luo, S Ulukus, A Ephremides, Optimal sequences and sum capacity of symbol asynchronous CDMA systems. IEEE Trans. Inf. Theory. 51(8), 2760-2769 (2005)

15. L Cottatellucci, R Muller, M Debbah, Asynchronous CDMA systems with random spreading - Part I: fundamental limits. IEEE Trans. Inf. Theory. 56(4), 1477-1497 (2010)

16. X Wang, HV Poor, Space-time multiuser detection in multipath CDMA channels. IEEE Trans. Signal Process. 47(9), 2356-2374 (1999)

17. A Yener, RD Yates, S Ulukus, CDMA multiuser detection: a nonlinear programming approach. IEEE Trans. Commun. 50(6) (2002)

18. Q Li, CN Georghiades, X Wang, Blind multiuser detection in uplink CDMA with multipath fading: a sequential EM approach. IEEE Trans. Commun. 52(1) (2004)

19. T Fulghum, D Cairns, C Cozzo, YP Wang, G Bottomley, Adaptive generalized rake reception in DS-CDMA systems. IEEE Trans. Wirel. Commun. 8(7), 3464-3474 (2009)

20. Y Cai, RD Lamare, Space-time adaptive MMSE multiuser decision feedback detectors with multiple-feedback interference cancellation for CDMA systems. IEEE Trans. Veh. Technol. 58(8), 4129-4140 (2009)

21. YH Yun, JH Cho, On sum capacity of continuous-time overloaded CDMA systems. Paper presented at Inf. Theory and Appl. Workshop (ITA), San Diego, CA, USA, 1-10 (2010)

22. J Ahmed, K Hamdi, Spectral efficiency of asynchronous MC-CDMA with frequency offset over correlated fading. IEEE Trans. Veh. Technol. 62(7), 3423-3429 (2013)

23. R Gray, On the asymptotic eigenvalue distribution of Toeplitz matrices. IEEE Trans. Inf. Theory. 18(6), 725-730 (1972)

24. W Hirt, J Massey, Capacity of the discrete-time Gaussian channel with intersymbol interference. IEEE Trans. Inf. Theory. 34(3), 38-38 (1988)

25. TM Cover, JA Thomas, Elements of Information Theory. (Wiley-Interscience, Hoboken, NJ, USA, 2006)

26. A Lapidoth, A Foundation in Digital Communication. (Cambridge University Press, UK, 2009)

27. S Verdú, Multiuser Detection. (Cambridge University Press, UK, 1998) 
28. RG Gallager, Information theory and reliable communication. (John Wiley \& Sons, New York, USA, 1968)

29. HV Poor, An Introduction to Signal Detection and Estimation. (Springer-Verlag, New York, 1994)

30. JG Proakis, Digital communications. (McGraw-Hill, New York, 1995)

31. TT Do, TJ Oechtering, SM Kim, P Gunnar, in Paper presented at 80th IEEE Veh. Technol. Conf. Capacity analysis of continuous-time timevariant asynchronous uplink wideband CDMA system (Vancouver Canada, 2014), pp. 1-5

32. R Ahlswede, in Proc. of the 2nd. Int. Symp. Infor. Theory (ISIT). Multi-way communication channels (Armenia, USSR, 1971), pp. 23-51

33. BM Hochwald, ST Brink, Achieving near-capacity on a multiple-antenna channel. IEEE Trans. Commun. 51(3), 389-399 (2003)

34. SM Kim, TT Do, TJ Oechtering, P Gunnar, On the Entropy Computation of Large Complex Gaussian Mixture Distributions. IEEE Trans. Signal Process. 63(17) (2015)

35. JM Wozencraft, IM Jacobs, Principles of communication engineering. (Wiley, New York, 1965)

\section{Submit your manuscript to a SpringerOpen ${ }^{\circ}$ journal and benefit from:}

- Convenient online submission

Rigorous peer review

- Immediate publication on acceptance

- Open access: articles freely available online

- High visibility within the field

- Retaining the copyright to your article 